\title{
Caractérisation \\ d'anticorps monoclonaux dirigés contre les virus de la peste bovine et de la peste des petits ruminants : identification d'épitopes conservés ou de spécificité stricte sur la nucléoprotéine
}

\author{
G. Libeau ${ }^{1}$ J. T. Saliki ${ }^{2}$ A. Diallo ${ }^{1}$
}

\begin{abstract}
Mots-clés
Peste bovine - Peste des petits ruminants - Anticorps monoclonal $\mathrm{N}$ ucléoprotéine - Technique radioimmunologique - Immunofluorescence Test ELISA - M orbillivirus - Diagnostic.
\end{abstract}

\begin{abstract}
Résumé
Vingt-neuf anticorps monoclonaux (ACM) dirigés contre les souches virales vaccinales RPV-RBOK et RPVL de peste bovine (RPV) et la souche PPRV N IG $75 / 3$ de la peste des petits ruminants (PPRV) ont été caractérisés par radioimmunoprécipitation (RIPA), immunofluorescence (IFI) et immunoenzymologie (ELISA). Vingt-sept d'entre eux étaient dirigés contre la nucléoprotéine $(\mathrm{N})$; deux AcM étaient spécifiques de la protéine de fusion $(\mathrm{F})$ et de la protéine de matrice $(M)$ du virus homologue. Pour ceux qui n'étaient pas précipitants, la réactivité au regard de la protéine de structure était confirmée par IFI et ELISA. La réactivité en IFI de ces AcM a permis de classer des souches RPV et PPRV d'origine géographique différente et de les comparer à deux autres morbillivirus, la rougeole (MV) et la maladie de Carré (CDV). L'AcM dirigé contre la M n'a pas indiqué de variations épitopiques au sein des souches PPRV et I'ACM anti-F1 a délimité un site unique sur l'ensemble des souches RPV et PPRV tout en les distinguant de MV et de CDV. Les AcM anti-N ont été purifiés, biotinylés et analysés par compétition réciproque au regard des souches RPV-RBOK et PPRV-NIG 75/1 utilisées comme antigène de l'ELISA. Ils ont défini sur la nucléoprotéine de ces virus respectivement 6 et 7 sites antigéniques. Sur l'ensemble des sites délimités, certains étaient uniques à RPV (2 sites) et d'autres à PPRV (3 sites). Les AcM qui les reconnaissaient ont permis de distinguer les deux virus sans ambiguïté. Q uatre sites se chevauchant sur les virus RPV et PPRV étaient conservés sur l'ensemble des morbillivirus et les sites restants étaient communs à 2 morbillivirus au moins. Trois AcM caractérisés dans cette étude sont de bons candidats pour des tests de diagnostic différentiel (Libeau G. et coll., 1994, Vet. Rec., 134: 300-304 ; Libeau G. et coll., 1995, Res. vet. Sci., 58: 50-55).
\end{abstract}

\section{INTRODUCTION}

Les anticorps monoclonaux (AcM) sont des outils performants pour l'étude et l'identification des agents pathogènes. Leur utilité provient de trois grandes caractéristiques : leur spécificité réduite à un seul épitope, leur homogénéité et la possibilité de les produire en grande quantité.

1. CIRAD-EMVT, Campus international de Baillarguet, BP 5035, 34032 Montpellier Cedex 1, France

2. Oklahoma Animal Disease Diagnostic Laboratory, Oklahoma State University, Stillwater, OK 74078-0622, USA
Ils ont été appliqués de façon systématique à l'analyse des différences antigéniques existant au sein des morbillivirus. Des batteries d'AcM ont permis d'étudier chaque membre du genre Morbillivirus : CDV (30), MV (6, 29, 36, 38, 44), RPV (5, 24, 26, $27,41,42$ ) et PPRV (35). Une variation antigénique plus importante que celle observée précédemment avec les anticorps polyclonaux a pu être décelée. Cette variation épitopique qui différencie les prototypes de chacun des virus du genre $(27,39)$ peut être discernée également, à un degré moindre, entre les isolats d'un même virus. Ceci a été démontré initialement au sein des souches RPV par des AcM préparés contre MV et $\operatorname{CDV}(30,38,39)$. De façon similaire, les AcM spécifiques font apparaitre parmi les souches 
RPV un certain nombre de différences parfois liées à leur pouvoir pathogène $(24,26,41)$. Dans cette étude, 29 AcM obtenus contre les souches vaccinales de peste bovine RPV-RBOK et RPVL et la souche virulente PPRV-NIG 75/3 ont été caractérisés.

\section{MATERIEL ET METHODES}

\section{Cellules et virus}

Des cellules de rein de singe (Vero R) ont été cultivées en milieu essentiel minimum de Eagle (EMEM), supplémenté avec 5 p. 100 de sérum de veau fotal (SVF) et des antibiotiques $(40 \mu \mathrm{g} / \mathrm{ml} \mathrm{de}$ gentamicine et $2,5 \mu \mathrm{g} / \mathrm{ml}$ d'amphotéricine B). Les souches virales suivantes ont été utilisées pour la production d'anticorps monoclonaux (AcM) : la souche vaccinale de peste bovine RPV-RBOK (31), utilisée à 98 passages sur cellules de rein de bovin (BK/98) et 2 passages sur cellules Vero (Vero/2) (24), ainsi que la souche sauvage PPRV-NIG 75/3 (13), utilisée à 6 passages sur rein d'agneau (LK/6) et 6 passages sur Vero (Vero/6).

Le test d'immunofluorescence indirecte (IFI) a été réalisé avec les souches virales suivantes : les souches vaccinales de peste bovine (RPV), RPV-RBOK et la souche lapinisée RPVL (18), neuf souches virulentes, India, Koweit, Arabie Saoudite, Yemen, Liban, Oman, Reedbuck, Egypte et RBT1 (24) ; les souches vaccinales de peste des petits ruminants (PPRV) PPRV-NIG 75/1 (11) ; les souches virulentes PPRV-NIG 75/2, NIG 75/3, Ghana, Accra, Ibri, Dorcas, Mielik, Sennar (24). Les souches de laboratoire de la maladie de Carré (CDV ; Onderstepoort) et de la rougeole (MV ; Edmonston) ont été incluses comme contrôle de la spécificité des AcM.

Les souches RPV-RBOK, PPRV-NIG 75/1 et NIG 75/3 ont été utilisées lors du test d'immunoprécipitation (RIPA).

\section{Anticorps monoclonaux}

Les AcM utilisés dans cette étude provenaient de plusieurs fusions pour lesquelles les souris avaient subi des protocoles d'immunisation différents. Les AcM ont été obtenus avec les souches RPVRBOK BK/98 et la souche PPRV-NIG 75/3 (LK/6, Vero/6). Les AcM obtenus avec la souche RPV-RBOK de spécificité anti-nucléoprotéinique ont permis de définir le profil antigénique des souches virales citées dans une publication précédente (24). Les AcM dénommés B11 et Y13 dont la spécificité était également anti-nucléoprotéinique (41) ont été gracieusement fournis par le Pr Yamanouchi. Les hybrides ont été sélectionnés pour leur activité anti-RPV ou PPRV par ELISA indirect ou par IFI. Les cellules positives ont été clonées par dilution limite et inoculées par voie intra-péritonéale à des souris $\mathrm{Balb} / \mathrm{c}$ préparées au pristane (2,6,10,14-tétraméthylpentadécane, Janssen Chemicals) afin d'obtenir l'ascite contenant les anticorps. Les AcM ont été purifiés à partir de l'ascite sur protéine A-sépharose (CL-4B, Pharmacia) selon une méthode décrite par Ey et coll. (12). Ils ont ensuite été biotinylés avec l'ester de biotine-XX-NH (Clontech Laboratories) selon la méthode de Shaw et coll. (37).

L'identité des AcM utilisés dans cette étude est résumée dans le tableau I.

\section{Préparation de l'antigène}

La souche RPV-RBOK, ou PPRV-NIG 75/1, a été récoltée et clarifiée à $2500 \mathrm{~g}$ pendant $25 \mathrm{~min}$. Le virus a ensuite été concentré sur une cassette Pellicon munie d'un filtre dont le seuil de coupure était
Tableau I

Identité des AcM utilisés dans cette étude

\begin{tabular}{|c|c|c|}
\hline $\begin{array}{l}\text { Numéro } \\
\text { de rang }\end{array}$ & $\begin{array}{c}\text { Nom } \\
\text { de l'AcM }\end{array}$ & $\begin{array}{c}\text { Virus } \\
\text { d'immunisation }\end{array}$ \\
\hline 1 & $\mathrm{IIH} 2$ & RPV-RBOK \\
\hline 2 & $48-5$ & RPV-RBOK \\
\hline 3 & $1-1$ & RPV-RBOK \\
\hline 4 & $51-5$ & RPV-RBOK \\
\hline 5 & $3-1$ & RPV-RBOK \\
\hline 6 & $5-1$ & RPV-RBOK \\
\hline 7 & $\mathrm{IH} 1$ & RPV-RBOK \\
\hline 8 & IVB2 & RPV-RBOK \\
\hline 9 & IVE4 & RPV-RBOK \\
\hline 10 & IE6 & RPV-RBOK \\
\hline 11 & $36-5$ & RPV-RBOK \\
\hline 12 & $13-5$ & RPV-RBOK \\
\hline 13 & $17-5$ & RPV-RBOK \\
\hline 14 & Y13 & RPVL \\
\hline 15 & B11 & RPVL \\
\hline 16 & $33-4$ & PPRV-NIG 75/3 \\
\hline 17 & $110-4$ & PPRV-NIG 75/3 \\
\hline 18 & $25-2$ & PPRV-NIG 75/3 \\
\hline 19 & $38-4$ & PPRV-NIG 75/3 \\
\hline 20 & $29-2$ & PPRV-NIG 75/3 \\
\hline 21 & 163 & PPRV-NIG 75/3 \\
\hline 22 & 131 & PPRV-NIG 75/3 \\
\hline 23 & $23-3$ & PPRV-NIG 75/3 \\
\hline 24 & $156-4$ & PPRV-NIG 75/3 \\
\hline 25 & $26-1$ & PPRV-NIG 75/3 \\
\hline 26 & $22-3$ & PPRV-NIG 75/3 \\
\hline 27 & 103 & PPRV-NIG 75/3 \\
\hline 28 & $19-6$ & PPRV-NIG 75/3 \\
\hline 29 & 128 & PPRV-NIG 75/3 \\
\hline
\end{tabular}

de $100 \mathrm{kDa}$ (Millipore Corp., Bedford, MA, USA). La suspension virale a été centrifugée sur un gradient de sucrose 20-60 p. 100 diluée dans du TNE (0,010 M Tris-HCl, 0,150 M NaCl, 1 mM EDTA, pH 7,4) à $100000 \mathrm{~g}$ pendant 90 min sur un rotor Beckman SW51. La bande correspondant au virus a été centrifugée à nouveau à $100000 \mathrm{~g}$ pendant $90 \mathrm{~min}$. Le culot viral obtenu a été resuspendu dans du TNE et gardé à $-70^{\circ} \mathrm{C}$. La concentration protéique a été mesurée par la méthode à l'acide bicinchoninique (40). Ce matériel a été utilisé pour l'immunisation des souris et l'ELISA indirect.

\section{Test ELISA}

Les plaques ont été sensibilisées et incubées pendant $1 \mathrm{~h}$ avec le virus purifié RPV-RBOK ou PPRV-NIG 75/1 dilué dans une solution saline $(0,01 \mathrm{M}$ phosphate : PBS). Elles ont été lavées avec du PBS contenant 0,05 p. 100 de Tween 20 puis incubées $1 \mathrm{~h}$ avec les surnageants de cultures d'hybrides (volumes $50 \mu \mathrm{l}$ ). Après une série de trois lavages, le test a été poursuivi par l'addition d'un conjugué dirigé contre les globulines totales de souris couplées à de la peroxydase (Dako) puis de son substrat, l'ortho-phénylène diamine en présence d' $\mathrm{H}_{2} \mathrm{O}_{2}$. Les isotypes ont été déterminés au moyen d'anticorps spécifiques de chaque sous-classe (Nordic Imm-Lab). L'absorbance a été lue à $492 \mathrm{~nm}$ sur un lecteur ELISA automatisé (Anthos Labtec Instruments). 


\section{Test IFI}

Les cellules Vero saines ou infectées par les souches RPV-RBOK et PPRV-NIG 75/1 ont été cultivées en plaques 96 cupulcs. Lorsque l'effet cytopathogène (ECP) est devenu évident (80 p. 100), les cellules ont été fixées par une solution à 80 p. 100 d'acétone à température ordinaire. Les plaques contenant le surnageant des hybrides et des clones ont été incubées pendant $1 / 2 \mathrm{~h}$, rincées au PBS 0,05 p. 100 Tween 20, puis additionnées de conjugué anti-IgG totales de souris fluorescent (Nordic Imm-Lab). Après rinçage, les plaques ont été examinées au microscope à fluorescence. La positivité a été estimée par une échelle graduée entre une croix (réaction faible) et trois croix (réaction forte).

\section{RIPA}

Lorsque les cellules Vero infectées par le virus ont révélé un ECP d'environ 20-40 p. 100, elles ont été rincées dans du PBS et incubées $4 \mathrm{~h}$ dans un milieu de sevrage composé d'EMEM sans méthionine. Après un nouveau rinçage et l'addition dans ce milieu de $10 \mu \mathrm{Ci} / \mathrm{ml}$ de méthionine ${ }^{35} \mathrm{~S}$ ] (Radiochemical Center, Amersham), l'incorporation de cet acide aminé radioactif par les protéines synthétisées de novo a été réalisée sur une période de $3 \mathrm{~h}$. La méthode RIPA a été appliquée, comme décrite précédemment (24). L'électrophorèse en gel de 12,5 p. 100 de polyacrylamide et en tampon Laemmli (20) a été réalisée dans des conditions dénaturantes.

\section{ELISA de compétition}

Dans cet ELISA (C-ELISA) les anticorps biotinylés ont été mis en compétition avec eux-même et les autres AcM non biotinylés. La compétition est apparue quand les anticorps ont reconnu le même épitope.

Les conditions utilisées dans ce test ainsi que les volumes, les tampons et les temps d'incubation ont été basés sur la méthode décrite pour la détection d'anticorps spécifiques anti-RPV (22). L'anticorps non biotinylé a été dilué au $1 / 3$ à partir d'une concentration de $500 \mu \mathrm{g} / \mathrm{ml}$ et incubé pendant $45 \mathrm{~min}$ à $37^{\circ} \mathrm{C}$. L'anticorps biotinylé a été additionné à la première dilution qui correspondait au maximum de compétition (70-100 p. 100) contre l'anticorps homologue non biotinylé. Le mélange des deux anticorps a ensuite ćtć incubé, aprc̀s une séric de trois lavages, avec la streptavidineperoxydase (Clontech Laboratories) et l'ortho-phénylène diamine. Le pourcentage d'inhibition d'un AcM a été calculé à partir de la densité optique (DO) de l'échantillon selon la formule suivante :

Pourcentage d'inhibition :

$100 \times$ (1-DO échantillon/DO contrôle)

La DO de l'échantillon était celle obtenue en présence de l'inhibiteur et la DO de contrôle était celle obtenue en l'absence d'inhibiteur. Une inhibition de plus de 50 p. 100 était considérée comme positive.

\section{RESULTATS}

\section{Spécificité des AcM}

Un total de 29 clones produisant des anticorps contre RPV et PPRV ont été caractérisés. Leur spécificité a été déterminée par RIPA en conditions dénaturantes. Les résultats d'un panel représentatif sont illustrés dans les figures $1,2,3$ et 4 . Parmi les 15 AcM anti-RPV, dix anticorps (48-5, 51-5, 3-1, 5-1, IH1, IVB2, IE6, $36-5, \mathrm{~B} 11$ et $\mathrm{Y} 13$ ) ont réagi avec la protéine structurale $\mathrm{N}$ de la $\begin{array}{lllllllll}1 & 2 & 3 & 4 & 5 & 6 & 7 & 8 & 9\end{array}$

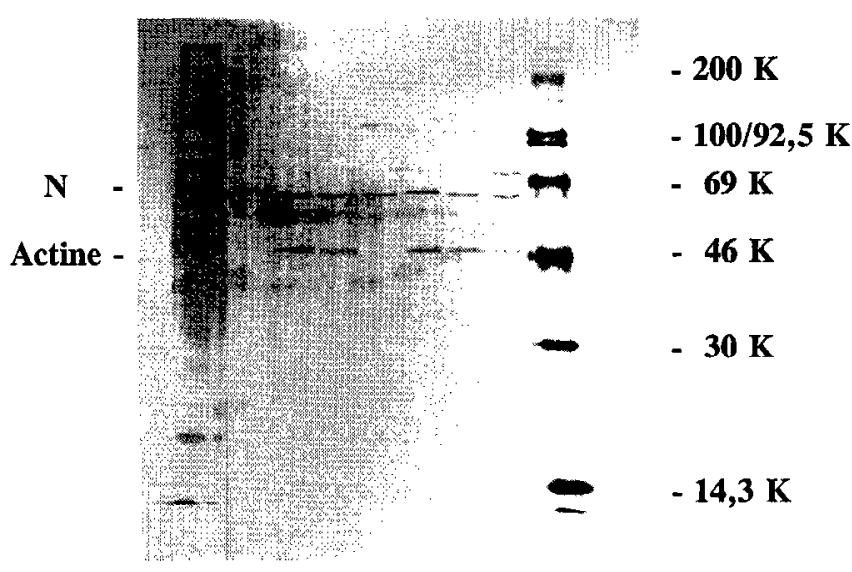

Figure 1 : analyse en SDS-PAGE de la protéine $N$ de la souche $R P V-R B O K$ après marquage à la méthionine $\left[{ }^{35} S\right.$ ] et immunopécipitation par les ACM anti-RPV. Ligne 1 : lysat intracellulaire de la souche RPV-RBOK. Lignes 2 à 7 : ACM 51-5; 48-5; IVB2; $3-1 ; I H 1 ; I E 6$. Ligne 8 : hyperimmunsérum anti-RBOK. Ligne 9 : marqueur de poids moléculaire.

(a)

(b)
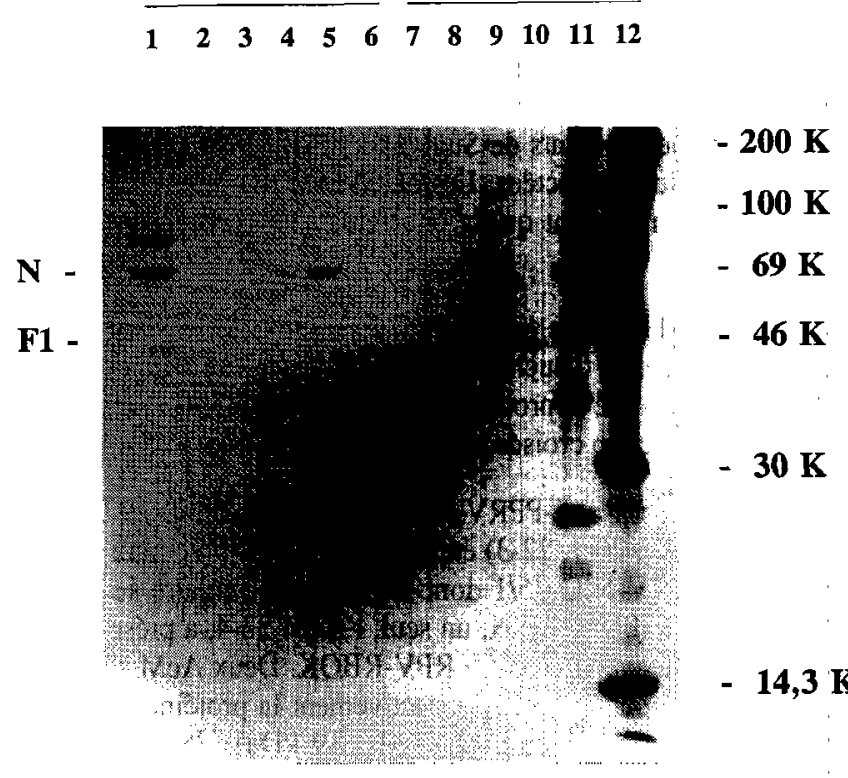

Figure 2 : réactivité de la protéine $N$ et $F 1$ de la souche $R P V$ RBOK de RPV (a) et de la souche PPRV-NIG 75/1 de PPRV (b) avec les ACM anti-N (lignes 3-5) et anti F1 (lignes 6 et 1.0). Les AcM utilisés en immunoprécipitation sont 36-5 (lignes 3 et 7), 5-1 (lignes 4 et 8), 33-4 (lignes 5 et 9), 128-1 (lignes 6 et 10). Ligne 7 : hyperimmunsérum anti-RBOK. Ligne 2 : ascite négatif. Ligne 11 : lysat intracellulaire de PPRV-NIG 75/1. Ligne 12 : marqueur de poids moléculaire.

souche RPV-RBOK dont le poids moléculaire se situe à $68 \mathrm{kDa}$ (figure 1). Cinq anticorps étaient incapables de précipiter cette protéine, cependant leur spécificité anti-nucléoprotéinique était déduite de leur réaction en IFI et ELISA (voir plus loin) (24). Il est à noter que l'immunisation avec la souche RPV-RBOK n'a produit que des AcM de spécificité anti-N. 


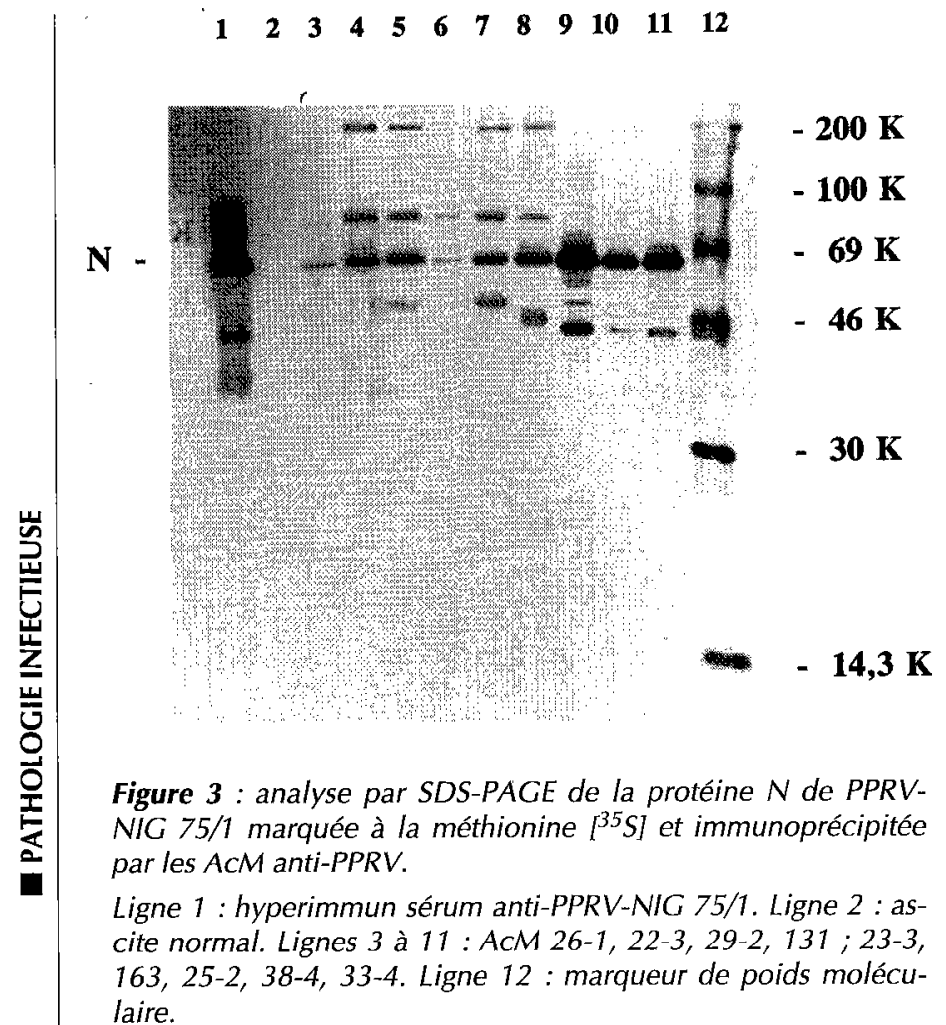
laire.

Les AcM B11 et Y13 inclus dans la présente étude étaient caractćrisćs commc étant spécifiques de la protéine $\mathrm{N}$ de la souche RPVL, selon les résultats de Sugiyama et coll (1989) en RIPA et en IFI sur cellules infectées. Les résultats d'immunoprécipitation de cette étude montrent qu'ils ont également reconnu la souche RPV-RBOK.

Parmi les AcM obtenus après immunisation avec les virus RPV, IE6, 13-5, 36-5, 5-1 (souche RPV-RBOK) et Y13 (souche RPVL) ont immunoprécipité la protéine $\mathrm{N}$ de PPRV-NIG 75/1. La figure 2 illustre cette réaction croisée pour deux d'entre eux, 36-5 et 5-1.

Sur quatorze AcM anti-PPRV, dix (33-4, 25-2, 38-4, 29-2, 163, 131, 23 3, 156-4, 26-1, 22-3) étaient capables de précipiter la protéine N de PPRV-NIG 75/1 dont le poids moléculaire se situe à $62 \mathrm{kDa}$ (figure 3). Parmi eux, un seul, l'AcM 33-4, a présenté une réaction croisée avec la souche RPV-RBOK. Deux AcM anti-PPR (128-1 et 19-6) ont précipité respectivement la protéine F1 (PM : $47 \mathrm{kDa}$ ) (28) et la protéine $\mathrm{M}$ (PM : $39 \mathrm{kDa}$ ) (28) (figure 4). L'AcM 128-1 a également réagi avec la protéine F1 de RPVRBOK ce qui corrobore les résultats indiquant une grande conservation de cette protéine parmi les morbillivirus $(28,39)$. Les résultats en RIPA d'un panel représentatif d'AcM obtenus après immunisation avec la souche PPRV-NIG 75/3 et réagissant avec RPV et PPRV sont illustrés dans la figure 2.

\section{Définition des sites antigéniques sur la protéine $N$ de RPV-RBOK et PPRV-NIG 75/1 par ELISA de compétition}

Vingt-deux AcM purifiés et biotinylés reconnaissant la protéine $\mathrm{N}$ de RPV et de PPRV ont permis de définir des sites antigéniques par compétition réciproque. La délimitation des sites antigéniques sur la souche RPV-RBOK et PPRV-NIG 75/1 sont respectivement représentés par les tableaux II et III. Chaque anticorps purifié est entré en compétition avec son homologue biotinylé. La réaction

\section{$\begin{array}{lllll}1 & 2 & 3 & 4 & 5\end{array}$}

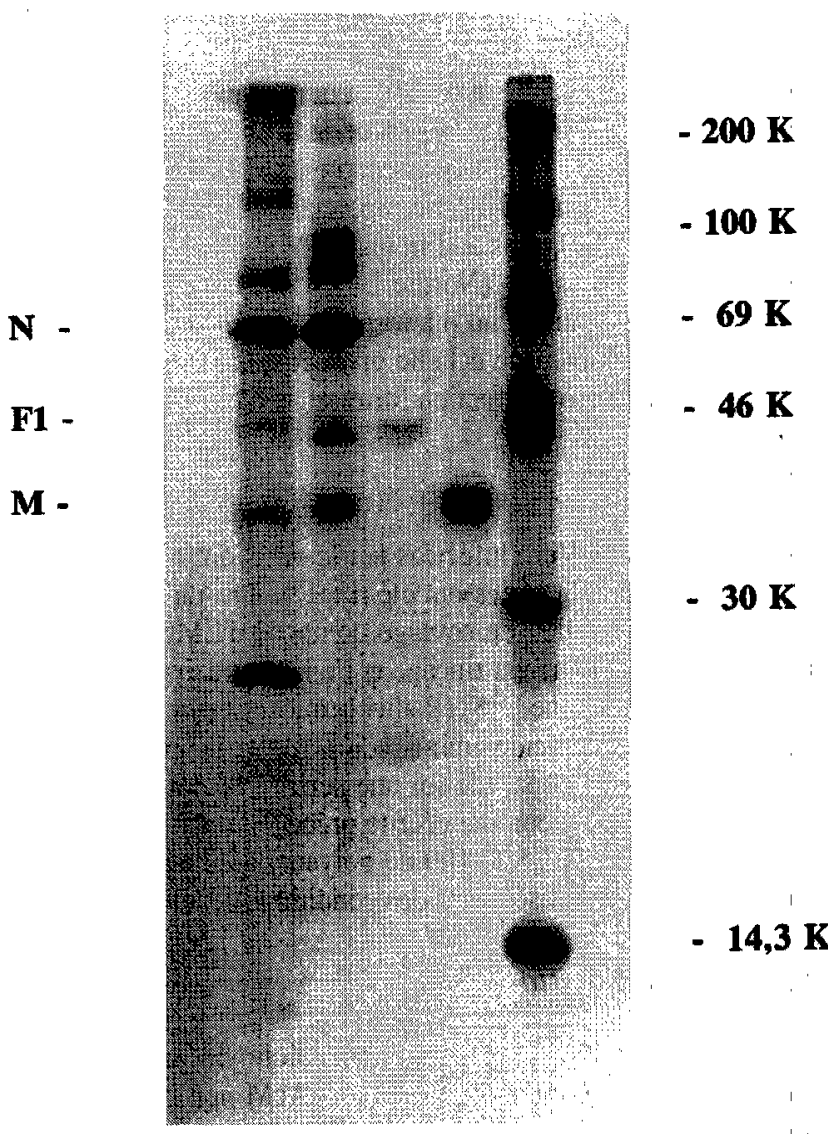

Figure 4 : analyse par SDS-PAGE des protéines $F 1$ et $M$ de PPRV-NIG 75/7 marquées à la méthionine ${ }^{35} S$ J et immunoprécipitées par les AcM 128-1 et 19-6.

Ligne 1 : lysat intracellulaire de PPRV-NIG 75/1. Ligne $2:$ hyperimmun sérum anti-PPRV-NIG 75/3. Ligne 3 : ACM 128-1. Ligne 4 : AcM 19-6. Ligne 5 ': marqueur de poids moléculaire.

n'était pas obligatoirement réciproque entre deux AcM du même site (par exemple 3-1 et 1-1). Les AcM 17-5 (anti-RPV), 156-4, 22-3 et 103 (anti-PPRV) étaient incapables d'entrer en compétition. l'AcM 26-1 est sorti de l'étude car il n'a pas reconnu la souche PPRV-NIG 75/1 en ELISA ni en IFI. Cet anticorps a cependant reconnu par ces tests, les souches PPR 75/2, 75/3 et Ghana. Les AcM dirigés contre la souche RPV-RBOK ont défini six sites antigéniques sur la protéine N. Par ailleurs, les sites II et III et $\mathrm{V}$ et VI se recouvraient partiellement (tablcau II).

Les AcM Y13 et B11 qui ont défini, à eux seuls, chacun un site, respectivement $V$ et IV sur la nucléoprotéine de RPVL (41), se sont retrouvés associés à d'autres AcM sur la nucléoprotéine de la souche RPV-RBOK. Y13 était associé à six autres AcM qui ont défini le site majeur IV. Ce site a induit une réponse immunitaire importante chez la souris mais également chez les bovins. En effet, deux de ces AcM, le IVB2 et le VE4, ont permis par CELISA, d'évaluer la présence d'anticorps après infection naturelle ou après vaccination. Bien qu'ils ne fussent pas neutralisants; ces anticorps avaient des titres en C-ELISA qui ont permis d'établir une corrélation significative entre ce test et celui de séroneutralisation (22). 

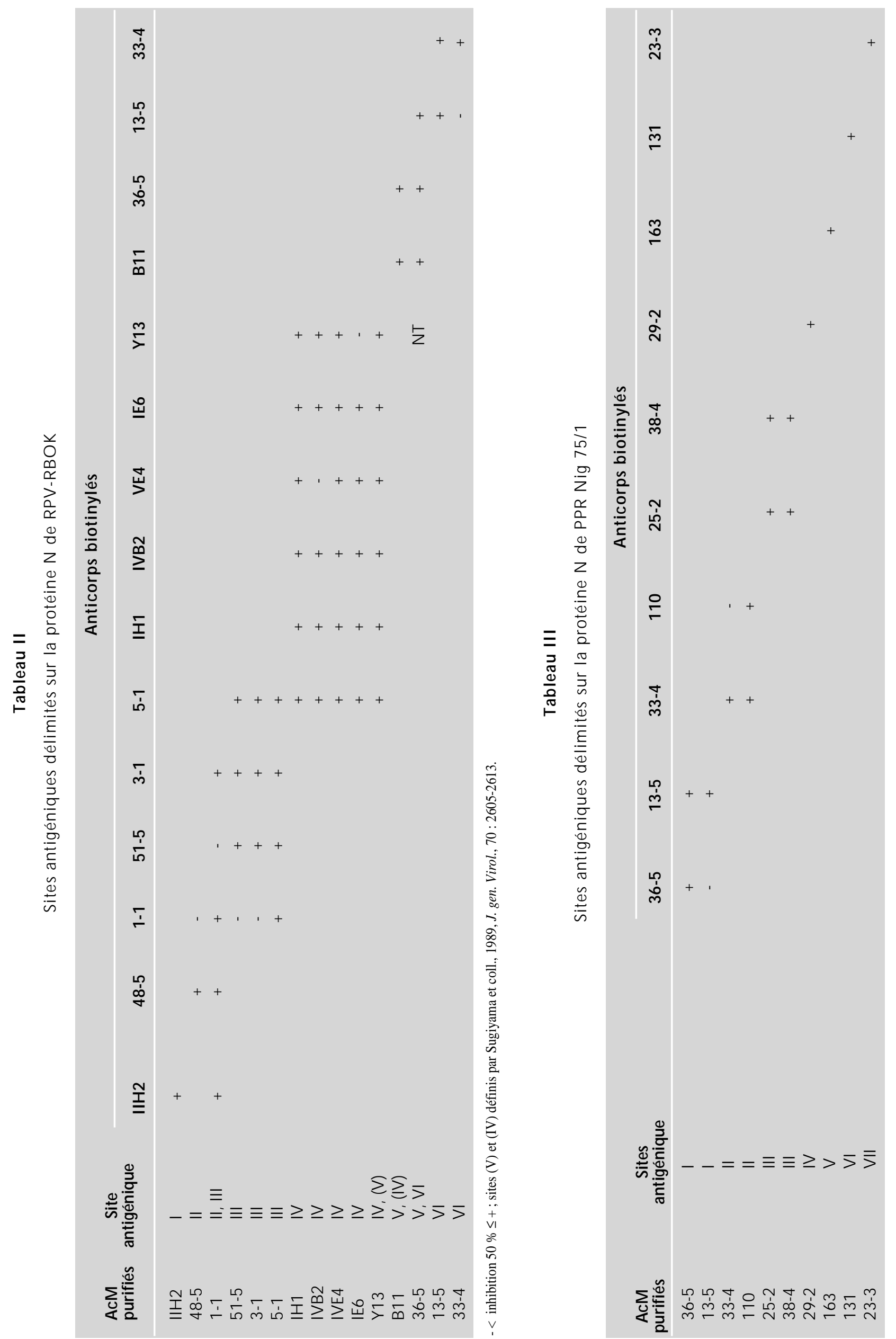
Les anticorps dirigés contre la souche PPRV-NIG 75/3 ont défini, sur la nucléoprotéine de la souche vaccinale PPRV-NIG 75/1, sept sites (tableau III). Chacun de ces sites a été défini par deux AcM au maximum qui, contre toute attente, étaient pour la plupart identiques au vu de leur réactivité en IFI avec les souches PPRV (tableau IV).

\section{Réaction croisée avec différentes souches de morbillivirus}

La réaction de 22 AcM (anti-N, F1 et M) a été testée en IFI sur quatre membres du genre morbillivirus (RPV, PPRV, MV et CDV) et plus particulièrement sur un éventail de souches RPV et PPRV d'origine géographique différente (tableau IV). Les AcM dirigés contre la nucléoprotéine ont pu être classés en trois groupes différents :

\section{Les AcM de spécificité restreinte :}

- spécifiques des souches vaccinales : un seul AcM, 51-5, site III, n'a réagi qu'avec deux souches vaccinales, RPV-RBOK et PPR PPRV-NIG 75/1, et la souche RPV-Inde, sans reconnaître aucune autre souche sauvage ;

- spécifiques de certaines souches : l'AcM 29-2, site (IV)*, n'a réagi qu'avec les souches PPRV-Nigeria et la souche PPRVGhana.

\section{Les AcM spécifiques d'un ou de plusieurs membres du genre :}

- sept AcM dirigés contre les sites antigéniques II, III, IV de la souche RPV-RBOK ont réagi exclusivement avec les souches RPV, sauf 5-1 (III) ;

- six AcM anti-PPRV dirigés contre les sites antigéniques (III), (V), (VI) et (VII) de la souche PPRV-NIG 75/1 ont réagi avec toutes les souches de PPRV. L'exclusivité de reconnaissance du groupe était valable pour 163 et 23-3.

3. Les AcM spécifiques de l'ensemble des morbillivirus : les AcM $36-5,13-5,33-4$ et 110-4 ont délimité une portion de la $\mathrm{N}$ commune à tous les morbillivirus. Deux sites antigéniques y étaient définis, les sites chevauchants V et VI sur RPV-RBOK ou distincts (I) et (II) sur PPRV-NIG 75/1.

L'AcM anti-F1 (128-1) a reconnu de façon uniforme les souches RPV et PPRV mais pas les virus MV et CDV.

L'AcM anti-M (19-6) a rattaché la souche RPV-Egypte à l'ensemble des souches PPRV. Il n'a reconnu aucune autre souche RPV. MV et CDV étaient également négatifs.

\section{DISCUSSION}

Une batterie de 29 AcM spécifiques des protéines N, F1 et M a été obtenue. Tous, sauf deux, étaient dirigés contre la nucléoprotéine. Les deux autres étaient de spécificité anti-F1 et anti-M. La prédominance des AcM anti-N, mis à part celle des AcM B11 et Y13 inclus dans l'étude, peut s'expliquer par les faibles doses d'antigènes utilisées qui, lors des protocoles d'immunisation, favorisent une réponse contre la protéine majoritaire : la nucléoprotéine (24). La méthode de sélection des hybrides semblait également orienter le type de spécificité : en ELISA, par exemple, si le virus entier est utilisé comme antigène, les protéines $\mathrm{F}$ et $\mathrm{M}$ sont sous-représentées par rapport à la $\mathrm{N}$; en IFI il est difficile de juger de la positivité de la fluorescence diffuse des glycoprotéines de surface ( $\mathrm{F}$ et $\mathrm{H})$ ainsi que de celle de la protéine $\mathrm{M}$.

* Les sites définis sur la souche PPRV-NIG 75/3 sont indiqués entre parenthèses.
Afin d'affiner les reconnaissances épitopiques de ces AcM, leur spécificité a été établie avec les souches virales RPV-RBOK, PPRV-NIG 75/1 et PPRV-NIG 75/3 par le test RIPA, et leurs réactions croisées ont été définies sur un ensemble de souches d'origine géographique différente, ainsi que sur MV et CDV par le test IFI. Il existe, bien évidemment, des différences de réactivité des AcM avec l'immunogène dans de telles études comparatives. Sheshberadaran et coll. (39), Libeau et Lefèvre (24) et Saliki (35) en ont donné des interprétations détaillées. Ces différences proviennent en majorité des traitements subis par l'antigène dans le test employé : le virion complet est fixé dans les tests ELISA et IFI, une protéine solubilisée réagit en RIPA. Dans la présente étude, parmi les 15 AcM anti-N RPV réagissant en IFI, seuls 10 ont été positifs en RIPA, et parmi les 14 AcM anti-PPRV (spécificité $\mathrm{N}, \mathrm{F} 1$ et $\mathrm{M}), 13$ ont réagi en IFI et 12 en RIPA.

Vingt-deux AcM dirigés contre la nucléoprotéine des virus RPV et PPRV ont permis, par C-ELISA, de délimiter des sites antigéniques. Six ont été définis sur la souche RPV-RBOK et 7 sur la souche PPRV-NIG 75/1. Pour certains des AcM biotinylés, l'encombrement stérique provoqué par le couplage a été responsable de son incapacité à entrer en compétition avec lui-même et les autres AcM testés. Une compétition non réciproque a également été observée dans les sites III, IV, VI ou (I) et (II). Elle était due à l'induction d'effets allostériques sur la protéine $\mathrm{N}$ lors de la fixation d'un AcM, qui rendait le site inaccessible à l'autre AcM. Cette dernière constatation a également été faite par Giraudon et coll. (15), Sugiyama et coll. (41) et Saliki (35). Sur les 13 sites délimités sur la nucléoprotéine, 5 ont permis d'établir une spécificité stricte RPV (sites II, IV) ou PPRV (sites (IV),(V), (VII)) des AcM qui les ont reconnus. Ces sites ont clairement établi l'identité distincte de RPV et PPRV dans le genre Morbillivirus, ce qui corrobore les résultats précédemment obtenus par Gibbs et coll. (14) et Diallo et coll. (9). Le site (IV) contenait un AcM de spécificité restreinte aux souches PPRV-Nigeria et à la souche Ghana $(\mathrm{GH})$. Le site III était défini par l'AcM 51-5, de spécificité apparemment restreinte aux souches vaccinales RPV-RBOK et PPRV-NIG 75/1 et à la souche RPV-Inde. La réactivité avec la souche PPRV-NIG 75/1 dépendait du test employé. En effet, il a réagi en ELISA indirect et non en IFI (22). De même, lors de son emploi dans un test C-ELISA, le pouvoir discriminatoire de cet AcM entre les anticorps d'origine vaccinale ou sauvage ne s'est pas confirmé (H. Unger, comm. pers.). Il est à noter que le site III incluait également des AcM de réactivité hétérogène (3-1 était spécifique des souches RPV et 5-1 a reconnu quelques souches de PPRV). Quatre sites se chevauchant deux à deux sur la nucléoprotéine de RPV et de PPRV ont été conservés sur l'ensemble des 4 morbillivirus étudiés (V, VI, (I), (II)). Les sites restants étaient communs à 2 morbillivirus au moins.

D'après le degré de similitude qui existait au niveau des protéines $\mathrm{N}, \mathrm{M}$ et $\mathrm{F} 1$, les isolats de chacun des virus RPV et PPRV étudiés ont pu être répartis en groupes ayant le même profil de réactivité. La comparaison des souches RPV a été par ailleurs décrite en détail dans une publication précédente (24). Les associations observées ont regroupé les souches selon leur proximité géographique et non pas selon leur pouvoir pathogène, comme certains auteurs ont pu le décrire (26). Par exemple, les souches RPV du MoyenOrient, dont faisaient partie RPV-Saoudi, souche hypervirulente, et RPV-Egypte, souche hypovirulente, avaient un profil antigénique très semblable. Parmi les souches PPRV, des différences antigéniques sont apparues pour $3 \mathrm{AcM}$ anti-RPV (IIH2, 36-5, 13-5) et 2 AcM anti-PPRV (110-4 et 29-2). Ils montrent que les souches originaires du Soudan et du Sultanat d'Oman formaient un groupe antigéniquement très proche qui se différenciait des souches du Ghana et du Nigeria. McCullough et coll. $(26,27)$ ont également 

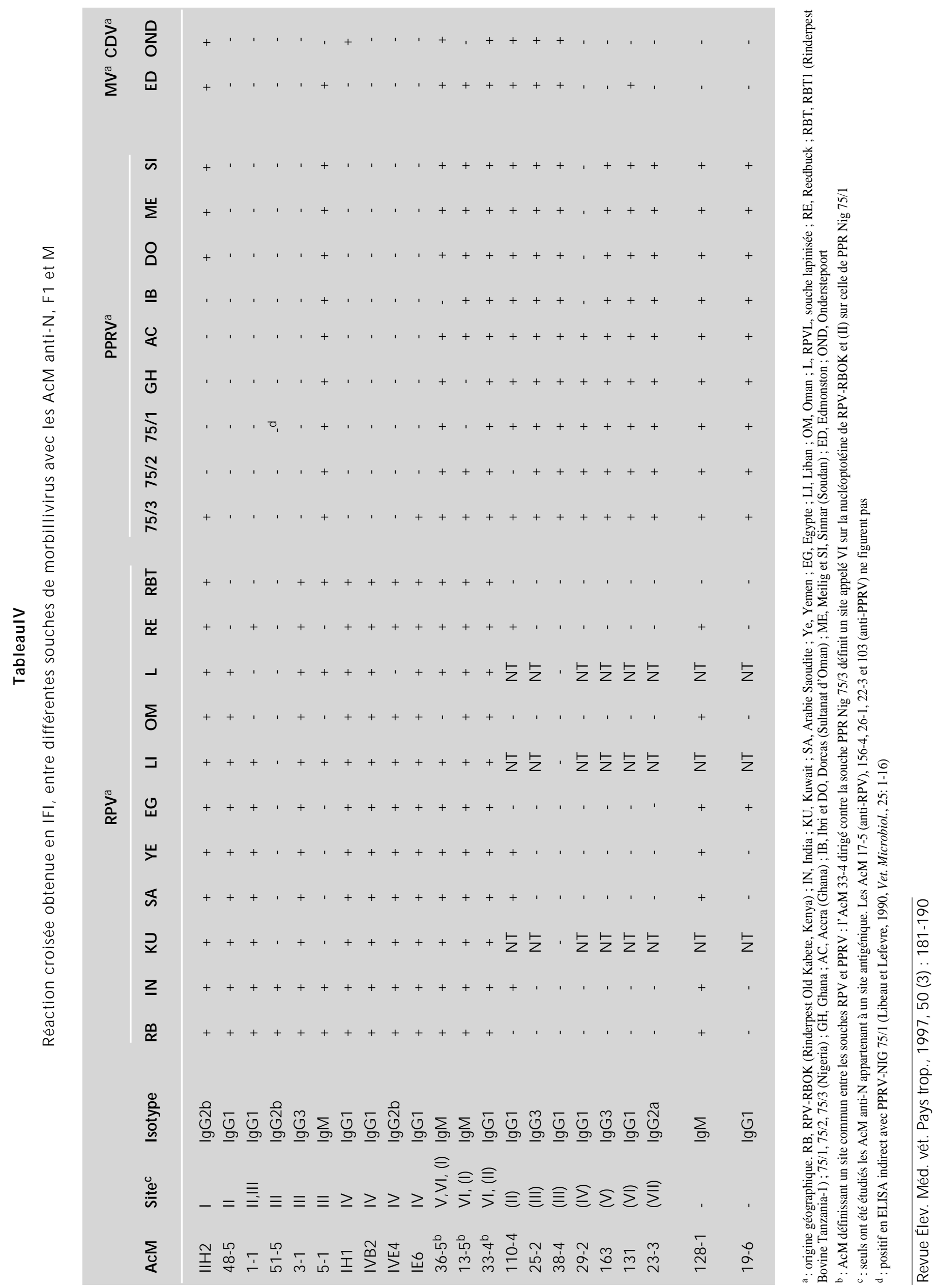
montré des variations épitopiques sur des souches PPRV à l'aide d'AcM dirigés contre les protéines internes de RPV. Saliki (35) a observé, à l'inverse, une réactivité uniforme de ces souches avec des AcM dirigés contre une protéine externe du virus PPRV, en l'occurrence la $\mathrm{H}$. Il conclut que cette uniformité de réaction est conforme à l'uniformité du pouvoir pathogène des souches de PPRV étudiées.

Avec d'autres méthodes d'analyse telle que l'électrophorèse en SDS, des différences significatives entre RPV et PPRV ont également été observées à l'examen des vitesses de migration de la protéine $\mathrm{N}$ et $\mathrm{M}$ de $\operatorname{RPV}(1,10)$ ou de la N de PPRV (43). Cette propriété a permis de proposer la protéine $\mathrm{N}$ comme marqueur biochimique pour la distinction entre RPV et PPRV (21).

Les études comparatives de séquences nucléotidiques montrent que la protéine $\mathrm{N}$ des morbillivirus est bien conservée sur les 400 premiers acides aminés, mais qu'il existe une partie variable hydrophile du coté C-terminal $(8,19,34)$. Ceci s'applique également pour quatre souches RPV bien étudiées - les souches vaccinales RPV-RBOK et virulentes Kabete « $\mathrm{O}$ », pour lesquelles l'identité est de 99 p. 100 sur les 400 premiers acides aminés avec les souches RPVL et RPV-Koweit. Cette homologie se réduit à 73,6 p. 100 (RPVL) et 79,2 p. 100 (RPV-Koweit) sur les 125 acides aminés restants (2). Il y a de fortes chances pour que les AcM capables de différencier deux souches d'un même virus soient dirigés contre l'extrémité C-terminale. Tel n'est pas forcément le cas d'un AcM capable de distinguer deux morbillivirus. En effet l'identité de séquence de la $\mathrm{N}$ entre différents morbillivirus est inférieure à 81 p. $100(2,33)$ (tableau V).

Le taux d'homologie entre les séquences des protéines $\mathrm{F}$ des différents morbillivirus est très élevé (autour de 70 p. 100) (17). A la fin de son processus de maturation, cette protéine est clivée en deux peptides F1 et F2 qui restent liés par un pont disulfure. L'extrémité amino-terminale du polypeptide F1 est la partie la plus conservée, probablement pour le maintien de la fonction particulière qui lui est attribuée. En effet, elle joue un rôle dans la fusion membranaire. Les réactions croisées observées du point de vue sérologique, mettent précisément en jeu cette extrémité N-terminale (32). Dans la présente étude l'AcM anti-F1 a également délimité un épitope unique pour l'ensemble des souches RPV et PPRV mais il était absent de CDV et MV. De plus, il est à noter que, grâce à sa forte homologie, le gène de la $\mathrm{F}$ peut être utilisé pour les études d'épidémiologie moléculaire des virus RPV et PPRV $(4,7,45)$.

\section{Tableau V}

Homologie de séquence entre les protéines codées par le génome de la souche vaccinale RPV-RBOK (RB) et celles issues de la souche sauvage parentale RPV-Kabete « $O$ » (RPV-K), de MV, CDV, du virus Sendai et de PPRV

\begin{tabular}{ccccrc} 
Protéine & \multicolumn{5}{c}{ Virus } \\
& RPV-K & MV & CDV & SeV & PPRV \\
N & 99,24 & 73,72 & 66,73 & 21,55 & NT \\
P & 99,40 & 60,52 & 47,01 & 13,54 & NT \\
C & 98,87 & 58,45 & 48,79 & 9,66 & NT \\
M & 99,14 & 88,54 & 77,65 & 35,82 & 70 \\
F & 98,94 & 77,92 & 66,08 & 28,27 & NT \\
H & 98,87 & 58,18 & 35,98 & 14,75 & NT \\
L & NT & 83,03 & 73,02 & 41,74 & NT
\end{tabular}

NT : non testé
La protéine de matrice $\mathrm{M}$ est la protéine la mieux conservée entre les morbillivirus $(2,3)$. Cependant, l'unique AcM anti-M obtenu a différencié les souches PPRV des souches RPV, MV et de CDV. Seule la souche RPV-Egypte a donné une réaction croisée avec les souches PPRV.

\section{CON CLUSIO N}

Il apparaît que les propriétés les plus intéressantes des AcM anti-N sont leur capacité de distinguer des souches PPRV de celles de RPV. Leur pouvoir distinctif important a été démontré également par Giraudon et coll. (15) et McCullough et coll. (26). Couplés ou non à la biotine, certains des AcM caractérisés dans cette étude sont utilisés dans des tests de diagnostic différentiel rapides utilisables en routine et applicables à des échantillons en grand nombre $(23,25)$.

\section{BIBLIO GRAPHIE}

1. ANDERSON E.C., HASSAN A., BARRETT T., ANDERSON J., 1990. O bservation of the pathogenicity for sheep and goats and the transmissibility of the strain of virus isolated during the rinderpest outbreak in Sri Lanka in 1987. Vet. Microbiol., 21: 309-318.

2. BARON M., BARRETT T., 1995. The sequence of the $N$ and $L$ genes of rinderpest virus, and the $5^{\prime}$ and $3^{\prime}$ extra-genic sequences: the completion of the genome sequence of the virus. Vet. microbiol., 44: 175-186.

3. BARON M., GOATLEY L., BARRETT T., 1994. Cloning and sequence analysis of the matrix $(M)$ protein gene of rinderpest virus and evidence for another bovine morbillivirus. Virology, 200: 121-129.

4. BARRETT T., AMAREL-DOEL C., KITCHING R.P., GUSEV A., 1993. $U$ se of the polymerase chain reaction in differentiating rinderpest field virus and vaccine virus in the same animals. Revue sci. tech. $0 \mathrm{ff}$. int. Epizoot., 12: 865-872.

5. BHAVANI K., KARANDE A.A., SHAILA M.S., 1989. Preparation and characterization of monoclonal antibodies to nucleocapsid protein $\mathrm{N}$ and $\mathrm{H}$ glycoprotein of rinderpest virus. Virus Res., 12: 331-348.

6. BIRRER M.J., BLOOM B.R., UDEM S., 1981. Characterization of measles polypeptides by monoclonal antibodies. Virology, 106: 381-390.

7. CHAMBERLAIN R.W, WAMWAYI H.M., HOCKLEY E., SHAILA M.S., GOATLEY L., KN O WLES N.J., BARRETT T., 1993. Evidence for different lineages of rinderpest virus reflecting their geographic isolation. J. gen. Virol., 74: 2775-2780.

8. DIALLO A., BARRETT T., BARBRON M., MEYER G., LEFEVRE P.C., 1994. Cloning of the nucleocapsid gene of peste des petits ruminants virus: relationship to other morbilliviruses. J. gen. virol., 75: 233-237.

9. DIALLO A., BARRETT T., BARBRON M., SUBBARAO S.M., TAYLOR W.P., 1989. Differentiation of rinderpest and peste des petits ruminants viruses using specific CDNA clones. J. Virol. Methods, 23: 127-136.

10. DIALLO A., BARRETT T., LEFEVRE P.C., TAYLOR W.P., 1987. Comparison of proteins induced in cells infected with rinderpest and peste des petits ruminants. J. gen. Virol., 68: 2033-2038.

11. DIALLO A., TAYLOR W.P., LEFEVRE P.C., PRO VOST A., 1989. Atténuation d'une souche de virus de la peste des petits ruminants: candidat pour un vaccin homologue vivant. Revue Elev. M éd. vét. Pays trop., 42 : 311-319. 
12. EY P.L., PROWSE S.J., JEN KIN C.R., 1978. Isolation of pure $\lg G 1$, $\lg \mathrm{g} 2 \mathrm{a}$, and $\lg \mathrm{G} 2 \mathrm{~b}$ immunoglobulins from mouse serum using protein $A$ Sepharose. Biochemistry, 15: 429-436.

13. FU RLEY C.W., TAYLOR W.P., O BI T.U., 1987. An outbreak of peste des petits ruminants in a zoological collection. Vet. Rec., 121: 443-447.

14. GIBBS E.P.J., TAYLOR W.P., LAWMAN M.J.P., BRYANT J., 1979. Classification of peste des petits ruminants as the fourth member of the genus Morbillivirus. Intervirology, 11: 268-274.

15. GIRAUDON P., JACQUIER M.F., WILD T.F., 1988. Antigenic analysis of African measles virus field isolates: identification and localisation of one conserved and two variable epitopes sites on the NP protein. Virus Res., 18: 137-152.

16. HAFFAR A., LIBEAU G., MOUSSA A., DIALLO A., The matrix protein of peste des petits ruminants virus alone is sufficient to promote the formation of Paramyxovirus like vesicles from recombinant baculovirus infected cells. (soumis pour publication)

17. HSU D., YAMANAKA M., MILLER J., DALE B., GRUBMAN M., YILMA T., 1988. Cloning of the fusion gene of rinderpest virus: comparative sequence analysis with other morbilliviruses. Virology, 166: 149-153.

18. ISHII H., YOSHIKAWA Y., YAMANOUCHI K., 1986. Adaptation of the lapinized rinderpest virus to in vitro growth and attenuation of its virulence in rabbits. J. gen. Virol., 67: 275-280.

19. KAMATA H., TSUKIYAMA K., SUGIYAMA M., KAMATA Y. YOSHIKAWA Y., YAMANOUCHI K., 1991. Nucleotide sequence of CDNA to the rinderpest virus mRNA encoding the nucleocapsid protein. Virus Gene, 5: 5-15.

20. LAEMMLI U.K., 1970. Cleavage of structural proteins during the assembly of the head of bacteriophage T4. Nature, 227: 680-685.

21. LeFEVRE P.C., DIALLO A., 1990. Peste des petits ruminants. Revue sci. tech. O ff. int. Epizoot., 9 : 951-956.

22. LIBEAU G., DIALlO A., CALVEZ D., LefEVRE P.C., 1992. A competitive ELISA using anti-N monoclonal antibodies for specific detection of rinderpest antibodies in cattle and small ruminants. Vet. Microbiol., 31: 147-160.

23. LIBEAU G., DIALLO A., COLAS F., GUERRE L., 1994. Rapid differential diagnosis of rinderpest and peste des petits ruminants infections using an immunocapture ELISA. Vet. Rec., 134: 300-304.

24. LIBEAU G., LEFEVRE P.C., 1990. Comparison of rinderpest and peste des petits ruminants viruses using anti-nucleoprotein monoclonal antibodies. Vet. Microbiol., 25: 1-16.

25. LIBEAU G., PREHAUD C., LANCELOT R., COLAS F., GUERRE L., BISH OP D.H.L., DIALLO A., 1995. Development of a competitive ELISA for detecting antibodies to the peste des petits ruminants virus using a recombinant nucleoprotein. Res. vet. Sci., 58: 50-55.

26. MCULLOUGH K.C., OBI T.U., SHESHBERADARAN H., 1991. Identification of epitope(s) on the internal virion proteins of rinderpest which are absent from peste des petits ruminants virus. Vet. microbiol., 26: 313-321.

27. MCULLOUGH K.C., SHESHBERADARAN H., N ORRBY E., OBI T.U., CROWTHER J.R., 1986. Monoclonal antibodies against morbilliviruses. Revue sci. tech. Off. int. Epizoot., 5: 411-427.

28. MEYER G., DIALLO A., 1995. The nucleotide sequence of the fusion protein gene of the peste des petits ruminants virus: the long untranslated region in the 5 -end of the F-protein gene of morbilliviruses seems to be specific to each virus. Virus Res., 37: 23-38.

29. NORRBY E., CHEN S.N., TOGASHI T., SHESHBERADARAN H., JOHNSON K.P., 1982. Five measles antigens demonstrated by use of the mouse hybridoma antibodies in productively infected tissue culture cells. Arch. Virol., 71: 1-11.
30. ORVELL C., SHESHBERADARAN H., N ORRBY E., 1985. Preparation and characterization of monoclonal antibodies directed against four structural components of canine distemper virus. J. gen. Virol., 71: 2085-2092.

31. PLOWRIGHT W., 1962. The application of monolayer tissue culture technique in rinderpest research. II. The use of attenuated culture virus as a vaccine for cattle. Bull. 0 ff. int. Epizoot., 57: 253-276.

32. RICHARDSON C.D., HULL D., GREER P., HASEL K., BERKOVICH A., ENGLUND G., BELLINI W., RIMA B.K., 1986. The nucleotide sequence of the mRNA encoding the fusion protein of measles virus (Edmonston strain): a comparison of fusion protein from several different paramyxoviruses. Virology, 155: 508-523.

33. RIMA B.K., WISHAUPT R.G.A., WELSH M.J., EARLE J.A.P., 1995. The evolution of morbilliviruses: a comparison of nucleocapsid gene sequences including a porpoise morbillivirus. Vet. microbiol., 44: 127-134.

34. ROZENBLATT S., EIZENBERG O., BEN LEVY R., LAVIE V., BELLINI, W.J., 1985. Sequence homology within the morbilliviruses. J. Virol, 53: $684-690$

35. SALIKI J.T., 1993. Structural and functional characterization of various isolates of peste des petits ruminants virus using monoclonal antibodies. PhD Diss., Cornell U niversity, Ithaca, NY, USA, 208 p.

36. SATO T.A., FUKUDA A., SUGIURA A., 1985. Characterization of major strucural proteins of measles virus with monoclonal antibodies. J. gen. Virol., 66: 1397-1409.

37. SHAW R.D., VO P.T., OFFIT P.A., COULSON B.S., GREENBERG H.B., 1986. Antigenic mapping on the surface of rhesus rotavirus. Virology, 155: 434-451.

38. SHESHBERADARAN H., CHEN S., NORRBY E., 1983. Monoclonal antibodies against five structural components of measles virus. 1. Characterization of antigenic determinants on nine strains of measles virus. Virology, 128: 341-358.

39. SHESHBERADARAN H., NORRBY E., MCULLOUGH K.C., CARPENTER W.C., ORVELL C., 1986. The antigenic relationship between measles, canine distemper and rinderpest viruses studied with monoclonal antibodies. J. gen. Virol., 67: 1381-1392.

40. SMITH P.K., KROHN R.I., HERMANSON G.T., MALLIA A.K., GARTNER F.H., PROVENZANO M.D., FUJIMOTO E.K., GOEKE N.M., OLSON B.J., KLENK D.C., 1985. Measurement of protein using bicinchoninic acid. Anal. Biochem., 150: 76-85.

41. SU GIYAMA M., MINAMOTO N., KINJO T., HIRAYAMA N., SASAKI H., YOHIKAWA Y., YAMANOUCHI K., 1989. Characterization of monoclonal antibodies against four structural proteins of rinderpest virus. J. gen. Virol., 70: 2605-2613.

42. SU GIYAMA M., MINAMOTO N., KINJO T., HIRAYAMA N., ASANO K., TSU KIYAMA-KOHARA K., YOSHIKAWA Y., YAMANOUCHI K., 1991. Antigenic and functional characterization of rinderpest virus envelope proteins using monoclonal antibodies. J. gen. virol., 72: 18631869.

43. TAYLOR W.P., AL BUSAIDY S., BARRETT T., 1990. The epidemiology of peste des petits ruminants in the sultanate of 0 man. Vet. microbiol., 22: 341-352.

44. TO GASH I T., O RVELL C., VARTDAL F., NORRBY E., 1981. Production of antibodies against measles virions by use of mouse myeloma technique. Arch. Virol., 67: 149-157.

45. WAMWAYI H.M., FLEM IN G M., BARRETT T., 1995. Characterisation of African isolates of rinderpest virus. Vet. Microbiol., 44: 151-163.

Reçu le 9.9.97, accepté le 3.11.97 


\section{Summary}

Libeau G., Saliki J.T., Diallo A. Characterization of monoclonal antibodies against rinderpest and peste des petits ruminants viruses: identification of shared or virus-specific epitopes on the nucleoprotein

Twenty-nine monoclonal antibodies (MAbs) prepared against two rinderpest virus (RPV) vaccine strains (RPV-RBOK and RPVL) and the NIG 75/3 isolate of peste des petits ruminants virus (PPRV) were characterized by radioimmunoprecipitation essay (RIPA), immunofluorescent antibody (IFA) staining and enzyme-linked immunosorbent assay (ELISA). Twenty-seven MAbs had specificity for the nucleoprotein ( $\mathrm{N}$ ); two recognized the fusion (F1) and the matrix (M) protein of the homologous virus. The specificity of MAbs that did not react with a structural protein in RIPA was determined by IFA staining and ELISA. Based on IFA reactivity, these MAbs were used to classify RPV, PPRV isolates of various origins and to compare them to two other morbilliviruses, measles virus (MV) and canine distemper virus (CDV). The anti-M MAb did not show any epitopic variations among PPRV isolates and the anti-F1 MAb delineated a single site on all RPV and PPRV isolates, which distinguished them from $M V$ and CDV. The anti-N MAbs were purified, biotinylated and analyzed by reciprocal competition using RPV-RBOK and PPRV-N IG 75/1 strains as ELISA antigens. They defined in the $N$ of each virus 6 and 7 antigenic sites, respectively. Among the delineated sites, some were specific to RPV (2 sites) and others to PPRV (3 sites). MAbs which recognized these specific sites were used to unequivocally differentiate between the two viruses. Four overlapping sites in RPV and PPRV were shared among all morbilliviruses and the remaining sites were common to at least 2 morbilliviruses. Three MAbs characterized in this study are good candidates for differential diagnostic tests (Libeau G. et coll., 1994, Vet. Rec., 134: 300-304 ; Libeau G. et coll., 1995, Res. vet. Sci., 58: 50-55).

Key words: Rinderpest - Pest of small ruminants - Monoclonal antibody - Nucleoprotein - Radioimmunoassay Immunofluorescence - ELISA - M orbillivirus - Diagnosis.

\section{Resumen}

Libeau G., Saliki J.T., Diallo A. Caracterización de anticuerpos monoclonales dirigidos contra los virus de la peste bovina y de la peste de los pequeños rumiantes: identificación de epítopes conservados o de especificidad estricta sobre la núcleo proteína

Se caracterizaron veintinueve anticuerpos monoclonales (ACM) dirigidos contra las cepas virales vaccinales RPVRBOK y RPVL de peste bovina (RPV) y de la cepa PPRV N IG $75 / 3$ de la peste de los pequeños rumiantes (PPRV), mediante radio inmunoprecipitación (RIPA), inmunofluorescencia (IFI) y immunoenzimología (ELISA). Veintisiete de éstos estaban dirigidos contra la nucleoproteína $(\mathrm{N})$; dos AcM fueron específicos a la proteína de fusión $(F)$ y la proteína de la matriz (M) del virus homólogo. Para aquellos que no fueron precipitantes, la reactividad con respecto a la proteína de estructura se confirmó mediante IFI y ELISA. La reactividad con IFI de estos AcM permitió clasificar las cepas de RPV y PPRV de diferente origen geográfico y compararlas con dos otros morbilivirus, rubiola (MV) y enfermedad de Carré (CDV). La ACM dirigida contra la M no presentó variaciones epitópicas dentro de las cepas PPRV y la AcM anti F1 resultó un sitio único sobre el conjunto de las cepas RPV y PPRV, al tiempo que se diferenció la MV y de la CDV. Las AcM anti-N se purificaron, biotinilaron y analizaron mediante competencia recíproca, con respecto a las cepas RPV-RBOK y PPRV-NIG 75/1 utilizadas como antígenos de ELISA. Estos definieron sobre la núcleo proteína de estos virus respectivamente 6 y 7 sitios antigénicos. Sobre el total de los sitios delimitados, algunos fueron unicamente RPV (2 sitios), otros PPRV (3 sitios). Las AcM que los reconocieron permitieron distinguir los dos virus sin ambigúidad. Cuatro sitios que se intercalaron sobre los virus RPV y PPRV, se conservaron sobre el conjunto de los morbilivirus y los sitios restantes fueron comunes al menos a 2 morbilivirus. Tres AcM caracterizados en este estudio fueron buenos candidatos para las pruebas de diagnóstico diferencial (Libeau G. et coll., 1994, Vet. Rec., 134: 300-304 ; Libeau G. et coll., 1995, Res. vet. Sci., 58: 50-55).

Palabras clave: Peste bovina - Peste de los pequeños rumiantes - Anticuerpo monoclonal - Nucleoproteína Técnica radioinmunologica - Inmunofluorescencia - ELISA Morbilivirus - Diagnóstico. 